\title{
Characteristics and prognosis of cardiac arrest victims cared by nurse firefighters in France
}

S. Lagadec ${ }^{1}$, J. Escutnaire ${ }^{2}$, V. Baert ${ }^{2}$, F.X. Laborne ${ }^{1}$, C. Bazzoli ${ }^{1}$, B. Garrigue ${ }^{1}$, M. Dhers ${ }^{3}$, H. Hubert ${ }^{2}$, G. Réac ${ }^{2}$.

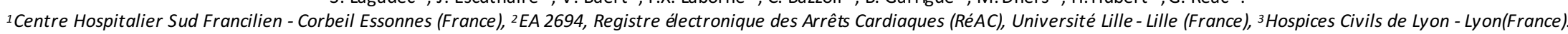

Purpose: Cardiac arrest (CA) victims' prognosis highly depends on how quickly advanced life support (ALS) is implemented. While waiting for mobile medical teams (MMT) to arrive on scene, some manoeuvres can be implemented by nurses which can be part of some firefighters' teams which of ten arrive before MMT in France. This study purpose is to characte rise patients which a re care d by firefighters' teams including a nurse and to compa re the ir outcome with patients cared without firefighter nurse.
Methods: National comparative multicentre study based on the French national cardiac arrest registry (RéAC) data gathere d be tween July 2011 and August 2016. Multivariate analysis of outcomes: ROSC, vital status at hospital admission, vital status at 30 days post-CA and neurological outcome. Comparison between CA cared by firefighter nurses and those cared by firefighters' teams without nurse's presence.

Results: We included 19380 patients among which 1641 (8\%) we re cared by a firefighter nurse. The emergency call number (15,18 or 112$)$ did not had any effect on the presence of firefighter nurse $(p=0.26)$. Nurses cared more often traumatic cardiac arrests, young people and CA occurring outside of home $(p<0.001)$. No-flow and low-flow durations were shorter $(p<0.001)$ and cum ulated epinephrine doses were higher $(p<0.001)$. The multivariate logistic regression showed lower ROSC (0.73[0.63-0.83], $p<0.001)$ admission survival $(0.76[0.65-0.88], p<0.001)$. There was no impact of the ir presence on day 30 survival but was associated with poorer neurological outcome $(0.67[0.45-0.97], \mathrm{p}=0.037)$.

Conclusion: Firefighter nurses of ten care younger, more traumatic and on public sites patients. These particularities, mainly traumatic cause, can explain their more pejorative outcome. 